\title{
DESCRIPCIÓN DE LA FORMA Y FUNCIÓN DE LA PARTICIPACIÓN DE LOS JÓVENES EN LAS ACTIVIDADES INSTRUMENTALES DE LA VIDA DIARIA
}

\author{
DESCRIPTION OF THE FORM AND FUNCTION OF THE PARTICIPATION OF YOUNG \\ PERSONS IN INSTRUMENTAL ACTIVITIES OF THE DAILY LIFE
}

\section{Rut Cristina Leegstra'; María Fabiana Cacciavillani²; Mariela Anderson ${ }^{3}$; Sergio Esteban Remesar $^{4}$; María Cecilia Prada ${ }^{5}$; Liliana Cristiani ${ }^{6}$; Maricel Berttoloto ${ }^{7}$; Marina Collavini ${ }^{8}$.}

\begin{abstract}
Resumen:
El siguiente artículo tiene como objetivo Identificar y describir la participación de los jóvenes en las actividades instrumentales de la vida diaria (AIVD) . Para describir la participación ocupacional de jóvenes en las actividades instrumentales de la vida diaria se hizo necesario no solamente indagar acerca de la participación, sino analizar las formas ocupacionales y funciones que las atraviesan. Se trabajó con una metodología mixta cuali-cuantitativa, con un diseño de tipo exploratorio descriptivo. La muestra quedó conformada por 34 jóvenes. Se identificaron y describieron formas variadas de participan y de función que atraviesan cada actividad. Los resultados revelaron que todos los jóvenes participan en actividades instrumentales de la vida diaria, sin embargo ninguno de ellos participa en todas las actividades que se incluyen dentro de las AIVD.

En la discusión se reflexiona acerca del lugar que ocupan las AIVD tanto en relación a su clasificación, a su desarrollo, como su fin. Y se comparten algunas de las dificultades que experimentamos en el desarrollo de la investigación relacionado a la construcción del instrumento de indagación, relevamiento de datos, clasificación y categorización.
\end{abstract}

\section{Palabras claves:}

Actividades instrumentales de la vida diaria, jóvenes, forma ocupacional y función ocupacional.

\begin{abstract}
Summary
The present article has as aim to identify and describe the participation of young persons in the instrumental activities of the daily life (IADL). To describe the occupational participation of young people in the instrumental activities of the daily life it became necessary not only to investigate the participation, but to analyze the occupational forms and functions that cross them.

We worked with a mixed cuali-quantitative methodology, with an exploratory design of descriptive the type. The sample was composed by 34 young persons. Varied forms of participation and functions that crossed each activity were identified and described. The results revealed that all young people's take part in instrumental activities of the daily life, nevertheless none of them takes part in all the activities that are included in the IADL.

In the discussion, it is thought over the place that the IADL occupy in relation to the classification, to the development, and to the aims. And the paper shares some of the difficulties that we experiment on during the development of the investigation related to the construction of the, instrument of compilation of information, report of information, classification and categorization.
\end{abstract}

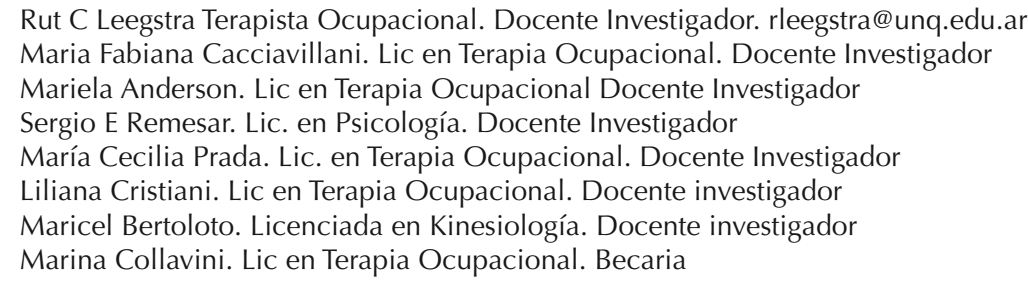




\section{Key words :}

Instrumental activities of the daily living, young people, occupational form and occupational function.

\section{INTRODUCCION}

El presente trabajo se desprende del proyecto de investigación "Participación Ocupacional en AVDI de jóvenes de diversos contextos sociales" Para el siguiente artículo se presentarán los resultados y conclusiones del primer objetivo específico: Identificar y describir la participación de los jóvenes en las actividades instrumentales de la vida diaria (AIVD).

La Organización Mundial de la Salud (OMS, 2001) y el Marco para la práctica de la Terapia Ocupacional (2008) utilizan el término participación para referirse al compromiso de una persona en situaciones vitales. Participar en una ocupación implica entonces tanto su desempeño como la experiencia subjetiva y su valoración.

Las Actividades Instrumentales de la Vida diaria (AIVD) son actividades de apoyo a la vida cotidiana en la comunidad y en el hogar. En su mayoría son co-ocupaciones ya que implican la presencia de otro en su realización y son sensibles a las necesidades e intereses de las personas que las realizan. Según la característica instrumental de estas actividades recae en su función auxiliar, de un instrumento o un medio para el logro de otras actividades.(Moruno Miralles 2006) Estas actividades están orientadas hacia la interacción con el medio, son más complejas que las actividades básicas como higienizarse ya que requieren de varios pasos y mayores habilidades cognitivas de organización y planificación.

La participación en estas actividades permite al sujeto mantener la independencia y vivir en la comunidad (Mulligan S.,2006; López Polonio,

9 Participación Ocupacional en AVDI de jóvenes de diversos contextos sociales .Universidad Nacional de Quilmas .Director Rut C.Leegstra . Codirector Sergio E Remesar .
2008; The American Journal of Ocupational Therapy, 2002 )

Entre estas actividades encontramos, el cuidado de mascotas, la crianza de niños, el cuidado de otros, gestión de la comunicación, movilidad en la comunidad, uso de la gestión financiera, gestión y mantenimiento de la salud, establecimiento y gestión del hogar, compras, preparación de comidas y limpieza, práctica de la religión, mantenimiento de la seguridad y responder a la emergencia (American Journal of Ocupational Therapy, 2008).

La participación en las AIVD en los jóvenes se condiciona por diversos factores que influyen en las elecciones ocupacionales y en el nivel de participación, tornándose determinantes en el desarrollo de la persona. Esto está asociado a la construcción de la identidad y el desempeño de los roles (Burak, 2001 ; Colmellas ,1986). Segun Kieljofner (2004), el desempeño de los roles ocupacionales influye en las ocupaciones, por un lado porque cada rol conlleva varias actividades y acciones propias de él, al mismo tiempo organizan las formas ocupacionales, de las ocupaciones y también el tiempo y los ciclos diarios y semanales y los horarios de las personas que ocupan varios roles en forma simultánea. Diversos autores hacen referencia a la influencia del contexto tornándose en facilitadores o barreras del grado y nivel de participación y en el significado atribuido a cada ocupación (Mulligan, 2006). El significado sólo pueden ser entendidos a la luz de los múltiples aspectos que influencian la elección y el nivel de participación ocupacional entre ellos la edad, el género, las expectativas y demandas familiares, sociales y culturales, el contexto histórico y político.

Para describir la participación ocupacional de jóvenes en las actividades instrumentales de la vida diaria se hace necesario no solamente indagar acerca de la participación en dichas activida- 
des, sino analizar las formas ocupacionales y funciones que las atraviesan.

Cuando se habla de formas ocupacionales se hace referencia a la manera de realizar una actividad, a las acciones específicas que se llevan a cabo en forma secuencial al participar en una actividad y que son sostenidas por el conocimiento colectivo y reconocible culturalmente. (Kielhofner 2004) La función de una ocupación se refiere a la forma en la que dicha ocupación influye en la adaptación de la persona.

\section{Metodologia}

\section{Objetivo: Identificar y describir la participación de los jóvenes en las actividades instrumentales de la vida diaria (AIVD)}

Se trabajó con una metodología mixta cualicuantitativa (Ynoub 2010; Kielhofner 2010) , con un diseño de tipo exploratorio descriptivo. (Hernández Sampieri, Fernández Collado, Baptista Lucio: 2010).

El muestreo no probabilístico. (Ynoub 2010; Hernández Sampieri, Fernández Collado, Baptista Lucio: 2010) quedó conformado por 34 jóvenes de entre 18 y 25 años de edad, de diferentes sexos y estratos sociales que residían o desarrollaban sus actividades en la zona de influencia de la Universidad Nacional de Quilmes.

Para la recolección de datos se creó un instrumento de indagación que fue puesto a prueba y ajustado.

Para la obtención de datos se realizaron entrevistas semi estructuradas construidas en base a los ejes de análisis propuestos en los objetivos de la investigación: participación en la AIVD, forma de participación y función. El cuestionario incluyo datos generales, participación en las AIVD, descripción de cómo participaba (forma ocupacional), para que participaba (función).

Se realizó el consentimiento informado a cada participante.
El análisis de los datos tomó en cuenta la participación de los jóvenes en las AIVD, la forma descripta, la función referida por los jóvenes. Se establecieron ejes de análisis para la creación de la matriz.

\section{RESULTADOS}

La muestra quedó conformada por 34 jóvenes de los cuales 18 eran mujeres y 16 hombres. En relación al estado civil de los jóvenes 22 eran solteros, 2 eran casados , 8 viviendo en pareja, 2 otra situación no especificada. Del total de los jóvenes 7 tenían hijos propios. En cuanto a la situación ocupacional de los entrevistados 10 estudiaban, 10 trabajan, 11 participaban en ambas actividades y 3 no trabajaban y no estudiaban.

La participación de los 34 jóvenes en las AIVD resultó : 11 jóvenes participaban en la actividad de cuidado de otros; 18 en cuidado de mascotas; 13 en crianza de niños; 34 en gestión de la comunicación; 34 en movilidad en la comunidad; 15 en uso de la gestión financiera; 23 en gestión y mantenimiento de la salud; 26 en gestión y establecimiento del hogar; 31 en preparación de comidas y limpieza; 8 en práctica de la religión; 30 en mantenimiento de la seguridad/emergencia y 29 en compras.

Descripción de las formas ocupacionales y función que atraviesan:

Cuidado de Otros: los 11 jóvenes que participaban nos refieren que lo realizaron cuidando a familiares (10) y a no familiares (3), con la función de supervisión y de atención que fue mencionada de la siguiente manera : "solo cuido a mi madre cuando todos salen, ya que ve poco" (P1) ${ }^{10}$; "Cuido a mi pareja, por ejemplo pidiéndole turno." (P5); "Cuido a mis hermanos." (P12); "Cuido a mi hermano de 12 cuando mi mamá no está." (P21); "Cuido al hijo de la vecina para colaborar." (P29);

10 P hace referencia al protocolo . 
"Cuido a mis hermanos, los superviso, les cocino." (P33).

En la ocupación de Cuidado de mascotas 18 jóvenes refirieron participar de dicha actividad. La forma de participación descripta por ellos es limpieza de excrementos, alimentación, baño y paseos. Lo expresaron de la siguiente manera: "Los alimento y limpio los excrementos."(P1); "Alimento al gato y al perro" (P2); "Le doy la comida." (8); "Los saco a pasear, les doy de comer." (9); "Le doy de comer, lo baño, lo paseo." (P16).

La Crianza de Niños fue mencionada por 13 jóvenes participando en actividades de ayudar con las actividades de alimentación, cuidado de la ropa, tareas del hogar y traslados y, jugando en todas estas actividades a los hijos (6) o a otros niños (8). Lo mencionaron de la siguiente forma : "cuido a mi hermano, en las salidas y el estudio, lo llevo y traigo de la escuela." (P 24); "cuido a mi hermano, le hago la comida, le plancho la ropa, y lo superviso." (P 19); "Cuido a mis hermanos." (P 27); "Cuido a mis primos." (P 30); "Los llevo a la escuela y ayuda a que hagan a tarea." (P34).

En relación a la función desempeñada, 6 jóvenes refieren participar en dicha actividad cuidando a sus propios hijos, como actividad vinculada a su rol materno, y 6 jóvenes participan en la actividad como una forma de colaborar con su familia.

La actividad de Gestión de la Comunicación fue referida por la casi totalidad, 34 jóvenes refirieron que participaban realizando llamadas con teléfono fijo (14), Ilamadas con teléfono móvil (31), a través de redes sociales (22), Mail (20), o de mensajes de texto (1).

En relación a la función de dicha actividad, todos los jóvenes que participan en la actividad lo hacen con funciones variadas, "Para buscar personas, buscar empleo y contactar amigos" (P11). De ellos 31 jóvenes la realizan con la finalidad de realizar actividades orientadas a la participación social. Refirieron: "Para comunicarme con amigos y familia"(P10); 7 jóvenes la realizan como un medio para desarrollar tareas vinculadas a la educación, 7 jóvenes la realizaban como un medio para realizar tareas de trabajo, y 1 refirió realizarla como un medio para las tareas de cuidado de sus hijos.

La Movilidad en la comunidad fue referida por 34 jóvenes quienes describieron su participación manejando auto (4), viajando en auto (4), tomando colectivos/micros (32), caminando (3), andando en bicicleta (7), andando en moto (2), tomando remis (9), tomando el tren (13).

En relación a la función con la que desempeñan dicha actividad, los jóvenes mostraron funciones variadas. Lo expresaron de la siguiente forma "al trabajo camino, a la cancha en colectivo." (P 3); "me traslado a trabajar y para ir al médico de mi mamá en colectivo o tren." (P 7). De los jòvenes entrevistados, 26 Jóvenes participan en la actividad de movilidad comunitaria como un medio para participar de actividades de participación social y de tiempo libre, 14 jóvenes la realizan como un medio para la actividad de educación, 16 jóvenes participan como un medio para la actividad de trabajo, 3 refieren participar en dicha actividad como un medio para la actividad de cuidado de otros.

15 jòvenes refirieron participar de la actividad de Uso de la Gestión Financiera. Refirieron que hacían compras con pagos financiados. "Compro ropa financiada con una extensión de la familia-" (P8); "Compro con tarjeta de crédito." (P11); "Compro cosas para a casa y ropa con tarjeta de crédito." (P 14); "Si , compro tele , ropa m, estufa." (P 25). La funciòn de dicha actividad no fue claramente determinada.

La Gestión y mantenimiento de la Salud fue señalada por 23 participantes como actividad en la que participaban, haciendo ejercicio físico (18), cuidándose en la alimentación (11); con visitas al médico (8), evitando riesgos y accidentes (3), tomado medicación (1).

Los jóvenes que participan en esta actividad la desempeñan con funciones variadas. Lo mencionaron así: "hago dieta, kinesiología, y al médico." (P2); "Voy al gimnasio y ando en bici." (P17). 6 jóvenes refirieron participar en actividades de deporte, gimnasia, que las realizan por placer personal, vinculadas a actividades de esparcimiento y 
tiempo libre, 1 con fines estéticos. 2 jóvenes refirieron participar en actividades de mantenimiento de la salud como un modo de cuidarse a si mismos vinculado al rol de madre o de planificación de la maternidad.

Gestión y establecimiento del hogar fue mencionado por 26 jóvenes quienes lo describieron realizando arreglos o ocupándose de ellos. Refirieron participar manteniendo y reparando (25), gestionando la reparación (12), o informando a otros del daño (2). "Reparo y mantengo mi ropa, recurro a terceros para problemas con las instalaciones del hogar." (P5), "Reparo la cama o el enchufe y si no se lo mando a hacer." (30), "Busco ayuda a ver quién puede arreglarlo." (P 31).

La actividad de Preparación de Comidas y Limpieza fue mencionada por 31 participantes quienes mencionaron: cocinando (28) o limpiando (25) de la siguiente forma: "Solo limpio, no cocino." (P 6), "Cocino a mi hijo y mantengo la casa." (P7); "Cocino y limpio si falta la señora de la casa." (P 9).

En relación a la función con la cual desempeñan la actividad: 21 jóvenes lo hacen como una forma de colaboración con la familia, 3 como una actividad de esparcimiento que realizan por placer personal, 4 refieren participar por necesidad, en un caso se desempeña como una actividad propia del rol de madre y en 1 caso se la vincula con la actividad de mantenimiento de la salud.

La actividad de Práctica de la Religión es referida en 8 casos participando de la siguiente forma: yendo a la iglesia (2). "Voy a la iglesia, al grupo de espiritualidad." (P2); "Voy a la iglesia dos veces por semana." (P4): "Voy a la Iglesia Evangélica." (P27) En dicha actividad no fue posible delinear la funcion con la que los jòvenes la desempeñan.

El Mantenimiento de la seguridad/emergencia mencionó en 30 casos. Los jóvenes refirieron formas variadas de participación en las que se colocaban el cinturón de seguridad, prendían las luces o miraban a ambos lados al cruzar la calle y en relación a la función pueden distinguirse la de prevenir el delito (en 24 casos), prevenir accidentes (11) o responder a la emergencia (2). Lo refirieron de la siguiente manera: "Cierro bien al salir y miro cuando guardo el auto." (P1), "Uso el cinturón de seguridad, miro bien a todos lados." (P3), "Uso cinturón de seguridad no ando con plata por la calle." (P14); "Cuido el hogar, prendo las luces y cierro la puerta." (P 17), "Estando atento a que no lo atropellen." (P32).

La actividad de compras es mencionada en 29 jóvenes de la siguiente manera: haciendo compras personales (24) o haciendo compras para el hogar (29). "Compro alimento." (P 6); "Compro ropa, comestibles, cosas que me pide mi madre." (P9); "Compro comida." (P10), "Compro cosas para la casa." (P10), "Voy al súper y compro cosas personales." (P19) .

En relación a la función de la actividad de compras en los 29 jóvenes que realizan compras para el hogar, el desempeño de esta actividad se vincula con la participación en las actividades de preparación de comidas y limpieza y gestión del hogar.

\section{COnClusiones}

Los resultados revelaron que, todos los jóvenes participan en actividades instrumentales de la vida diaria, sin embargo ninguno de ellos participa en todas las actividades que se incluyen dentro de las AIVD. Así, la casi totalidad de los jóvenes participan en la gestión de la comunicación, establecimiento y gestión del hogar, movilidad en la comunidad, compras, y en altísimos porcentajes del mantenimiento de la seguridad, preparación de comidas y limpieza. La menor participación se registró en la práctica de la religión.

Casi la totalidad de los jóvenes refirieron participar en las actividades de gestión de la comunicación y movilidad en la comunidad. Ambas actividades son desempeñadas como un medio para apoyar la participación en otra ocupación, como actividades sociales, educativas, de trabajo o de cuidado de los hijos.

Gestión y establecimiento del hogar y Preparación de Comidas y Limpieza son ocupaciones que 
parecieran estar relacionadas a la casi totalidad de los jóvenes de la comunidad, aunque la forma descripta se diversifique especialmente en relación a quienes las realizan y quienes las supervisan. También la participación en Compras es mencionada por porcentajes muy elevados. Es difícil imaginar que alguien no compre y acá nuevamente encontramos que describen la participación en actividades destinadas a sostener el desarrollo de otras ocupaciones, generalmente relacionadas al rol de madre, y a la gestión y mantenimiento del hogar y preparación de comidas y limpieza.

El Mantenimiento de la seguridad/emergencia también es una actividad en la que refieren participar la casi totalidad de los jóvenes describiendo diversas forma relacionada a prevención de accidentes o respuesta a una situación de emergencia. Esta actitud puede relacionarse al la vida en la zona metropolitana.

De las actividades relacionadas al cuidado de otros, de niños o de mascotas cabe destacar que es superior la participación registrada en cuidado de mascotas que la participación en la crianza de niños y cuidado de otros. La formas ocupacional descripta en la actividad de cuidado de mascotas está relacionada a los cuidados básicos de alimentación, limpieza de excrementos, baño, paseos, mientras que al indagar sobre el cuidado de otros los jóvenes sólo mencionaron a quienes cuidaban (familiares o no familiares) y la función de dicha actividad es de atención o supervisión. En relacion a la actividad de crianza de niños la forma descripta se diversifica a actividades de cuidado básico, actividades instrumentales que sostienen la crianza de los niños, nuevamente se menciona en la forma los destinatarios familiares o no familiares con funciones relacionadas directamente al rol de padres o de apoyo a la crianza.

Al indagar sobre la participación en el uso de la gestión financiera, los jóvenes solo mencionaron la realización de compras en cuotas, lo cual no permite distinguir una función ocupacional que la atraviese.

Por último, cabe destacar la participación en la Práctica de la Religión. Es interesante que esta actividad solamente es referida como la asistencia a actividades religiosas formales, como ir a la misa o templo pero otras formas de práctica de la religión no son mencionadas como podría ser rezar. Pareciera que otras formas de religiosidad no fueran enmarcadas en esta pregunta. La función ocupacional no ha podido descifrarse de los datos obtenidos de las entrevistas.

\section{DISCUSION}

La ocupación es un fenómeno complejo y difícil de estudiar por la diversidad de factores que intervienen y por la íntima relación con los diversos contextos.

El concepto, la idea, que las personas tienen acerca de las ocupaciones, y las formas y funciones ocupacionales varían a lo largo de las diferentes etapas de la vida relacionando estos aspectos con la dimensión temporal.

A lo largo del desarrollo de esta investigación se han presentado diversos cuestionamientos y dificultades. En primer lugar acerca del lugar que ocupan las AIVD tanto en relación a su clasificación a su desarrollo, como su fin. En segundo lugar en relacion a las dificultades experimentadas en el desarrollo de la investigación relacionado a la construcción del instrumento de indagación, relevamiento de datos, clasificación y categorización.

Las AIVD pueden presentarse en diversas formas y cumplir diferentes funciones simultáneamente. Es posible encontrar dos o más funciones en el desarrollo de una misma forma ocupacional. Por ejemplo si una abuela está con su nieto bañando al perro podría ser que está realizando actividades de crianza de niños o de cuidado de mascotas o de juego. ¿O será de todas ellas a la vez?

Por otro lado cabe la pregunta, ¿la simultaneidad en las que se presenta el desarrollo de las AIVD estará relacionada a las dimensiones históricas y sociales que afectan a las personas? Cocinar y hablar por teléfono. Trabajar y participar de espacios sociales en el campo virtual. 
Identificar el fin que atraviesan la actividad de uso de gestión financiera y práctica de la religión resulto dificultoso, debido a distintos motivos. Por un lado a la forma en cual se indagó y posiblemente a la confección del instrumento de indagación utilizado pero principalmente porque la función pareciera estar implícita en la misma clasificación. Esta situación se presenta en otras actividades donde la clasificación ya implica la función.

Es interesante destacar el hecho de que las AIVD pueden actuar como un medio para el desempeño de otras ocupaciones y la significatividad atribuida por la persona, como fue el caso de movilidad en la comunidad con el fin de participar del desarrollo de otra ocupación.

Si se piensa que las actividades actúan como un medio para el desempeño de otras (que se admiten como más significativas), se podría entonces establecer una jerarquía entre las ocupaciones que coloca a algunas AIVD como un medio para el logro de otras actividades como educación, trabajo y participación social.

Sin embargo, esta jerarquía no puede pensarse como tal si se tiene en cuenta que la participación en las ocupaciones, y la forma y función ocupacional que las define es subjetiva y está atravesada por factores contextuales, y dimensiones temporales.

Al mismo tiempo podría pensarse entonces que cualquier actividad, incluso las de trabajo y educación podrían ser un medio para el logro de otros fines, no siendo entonces un fin en si mismas. Desde un punto de vista subjetivo todas las actividades pueden estar destinadas a servir de un medio para el logro de fines superiores y, ninguna actividad constituye un fin en sí misma, ni es significativa por sí misma, sino que está encuadrada en una determinada cultura que le otorga valor y significado y estos significados pueden varias de una persona a otra.

El propósito o fin con el que la persona participa en una ocupación está influenciado por los roles que esta desempeña y por el significado que le atribuya a las ocupaciones.
Las AIVD al igual que todas las actividades adquieren su verdadero significado cuando están ligadas al entramado de actividades que la persona realiza, sus roles ocupacionales desempeñados y su historia personal.

En relación a las dificultades experimentadas durante el desarrollo de la investigación:

Esta investigación partió de una clasificación y definición establecida de las AIVD, sinembargo se cuestiona si hay otras actividades que podrían haber sido incluidas.

La construcción del instrumento de indagación fue un paso complejo, el mismo fue puesto a prueba y modificado en varias ocasiones debido a dificultades de comprensión de términos, especialmente en algunos contextos.

El entrenamiento en la toma fue realizado por los mismos investigadores del equipo pero requirió ajustes para no inducir posibles respuestas.

La diversidad de formas en que se presenta cada AIVD también generó dificultades al momento de relevar los datos. Preguntar por la participación en la preparación de comidas y luego por la práctica de la religión género un salto importante de temas.

Por otro lado dado, la diversidad de funciones en las que se puede presentar la misma actividad también implicó al encuestador pensar en el lugar en que clasificaría la misma haciendo difícil categorizar la participación en una ocupación. Por ejemplo andar en bicicleta puede pensarse como una actividad de esparcimiento, mantenimiento de la salud, movilidad en la comunidad. ¿El hecho de tomarla como una actividad instrumental de la vida diaria no presupone en si una determinada función?

La complejidad al investigar la participación ocupacional tanto por su extensión como por los aspectos que influyen en ella generó cuestionamientos en el seno del equipo de investigación que abre puertas a la discusión y desarrollo de nuevas investigaciones.

Se espera que los resultados expresados así como la discusión sean de utilidad para comprender de manera más completa la complejidad del 
fenómeno de la participación de los jóvenes en las AIVD y permita implementar estrategias de intervención en diversos proyectos y programas de salud, educación y comunidad en el partido de Quilmes así como también en poblaciones de situaciones contextuales similares.

\section{BIBLIOGRAFIA}

Acosta, N.; Caro, D.; Moran, D. (2008) Caracterización de las competencias transculturales del terapeuta ocupacional en su trabajo con grupos étnicos en un contexto urbano. Colegio mayor Nuestra Señora del Rosario. Facultad de rehabilitación y desarroIlo humano. Programa de terapia ocupacional. Colombia. (pág. 1-161).

American Journal of Ocupational Therapy (2008) Marco de Trabajo para Práctica en Terapia Ocupacional. Dominio y proceso. 2 da. Edición. Estados Unidos. Traducción española 2009 : (pág 2-12; 69)

Burak Solum Donas (2001) Adolescencia y juventud en América Latina. Ed. Libro Universitario Regional ,Costa Rica. (pág. 23-74)

Case-Smith, J. (2005) “Occupational Therapy for children" Editorial Elsevier Mosby. Estados Unidos . (pág. 521-526)

Christiansen C. The Eleanor Clarke Slagle Lecture.( 1999) Defining lives: occupation as identity: an essay on competence, coherence, and the creation of meaning. American Journal of Occupational Therapy. Estados Unidos .53:547-588

Colmellas, Maria Jesús (1986) Como medir y desarrollar los hábitos personales. Ed CEAC ,SA, Barcelona España .(Pág 9-36)

Crepeau .Cohm . Schells (2005) Willard \& Spakman Terapia Ocupacional 10ma ediciónPanamericana , Bs As , Argentina . (pág 97-108 ).

Hernández Sampieri R, Fernández Collado C, Baptista Lucio, P (2010) Metodología de la Investigación. Quita edición. México: McGrAw Gill Interamericana Editotres S.A.DE C.V.
Iwama, M. (2003) Toward Culturally Relevant Epistemologies in Occupational Therapy" The American Journal of Occupational Therapy, 57 (5): 582-588

Kielhofner G. (2006) Research in Ocupational Therapy . Methods of Inquiery for enhaciong practice. USA. Editorial FA Davis Company.

Kielhofner G. (2004) Conceptos fundamentales de Terapia ocupacional. Ed Davis Co , Estados Unidos . (pp 147-156)

Larson, R. W., \& Verma, S. (1999). How children and adolescents spend time across the world: Work, play, and developmental opportunities. Psychological Bulletin, 125,701-736

Law, M. (2002) Participation in the Occupations of Every Life. The American Journal of Occupational Therapy. 56 (6)640-648

Moruno, P., Romero D. (2006) “Actividades de la Vida Diaria". Editorial Elsevier Masson. España. (pág.3-21; 23-34; 35-50)

Mulligan Sherley (2003) "Terapia Ocupacional en Pediatría. Proceso de evaluación.". Editorial Panamericana. España. (pág 103-115; 147-163)

Municipio de Quilmas "Censo Social de Quilmes "2010 disponible: http://www.quilmes.gov.ar/desarrollo_social/descargas/sds_censo_social_2010.pdf. Fecha de última consulta: julio de 2012.

Organización Mundial de la Salud (2001) Clasificación internacional del funcionamiento, de la discapacidad, y de la salud. Génova (pág 3 -19)

Polonio López, Castellanos Ortega, Viana Moldes (2008) Terapia ocupacional en la Infancia. Teoría y Práctica. Editorial Médica Panamericana. España. (pág 121-134)

Samaja J (2008) Epistemología y Metodología. Elementos para una teoría de la investigación científica. Buenos Aires: Eudeba.

Hernández Sampieri R, Fernández Collado C, Baptista Lucio, P (1997) Metodología de la Investigación. México: McGrAw Gill Interamericana

Universidad Nacional de General Sarmiento/ UNICEF. Finalización de Estudios y Vuelta a la Escuela. Conurbano (Argentina). Disponible en: http://www.unicef.org/argentina/spanish/FINES_OKb. pdf . Fecha de última consulta: mayo de 2013.

Ynoub, R. (2010) "El diseño de investigación: una cuestión de estrategia" .Inédito. UNLA .Maestría en Metodologías de la Investigación Científica .Modulo III. Metodología de la Investigación. 